\title{
Microscopy101
}

\section{A Quick and Simple Technique for Orientating Diatoms for SEM and Light Microscopy}

\author{
Mark Webber ${ }^{1, *}$ and Elaine Humphrey ${ }^{2}$ \\ ${ }^{1} 455-2555$ Cook Rd., Galiano Island, British Columbia, Canada, V0N1P0 \\ ${ }^{2}$ Advanced Microscope Facility, University of Victoria, PO Box 1700 STN CSC, Victoria, British Columbia, \\ VSW 2Y2 Canada
}

*mwebber108@yahoo.ca

\begin{abstract}
A method was developed to expedite the imaging of diatoms by optimizing their orientation. This quick technique puts grooves into a SEM stub so that as the diatoms are laid down they either land on the flat surface or in a variety of positions in the grooves, thereby reducing time required to capture taxonomically critical features.
\end{abstract}

Keywords: Diatoms, imaging, orientation method, scanning electron microscopy, light microscopy

\section{Introduction}

Diatoms are unicellular eukaryotic microalgae that play important ecological roles on a global scale. Diatoms are responsible for $20 \%$ of global carbon fixation and $40 \%$ of marine primary productivity. Thus they are major contributors to climate change processes and form a substantial basis of the marine food web [1]. Over the last few decades, the first author on this article, Mark Webber, has collected samples of fresh water and marine diatoms from all over the world for taxonomic and ecological research. To identify diatoms to the genera or species level has required the development of novel and quick methods for orientating specimens for scanning electron microscopy (SEM) imaging, especially when a limited number of specimens are available. Taxonomic and other critical information can only be derived from variable orientations.

When preparing diatoms for microscopy they usually settle on slides, dishes, and stubs in either the valve or girdle views, which is seldom an optimal orientation for imaging (Figure 1) or taxonomy. It can take considerable time to find cells in a favorable view for the requirements of the research. Tilting the scanning electron microscope (SEM) stage does not always provide a sufficiently steep angle for the optimal imaging of critical features. Similarly, a light microscope (LM) examination of a limited number of specimens can leave important features obscured from view. Occasionally, with enough cells, one is fortunate that a cell has settled in an advantageous inclination. To enhance the chances of optimal positioning occurring, we present a procedure of placing grooves on SEM stubs with a diamond scribe at a steep enough angle for the viewing of diatoms. This often simplifies the SEM tilting and rotation procedure and minimizes the requirement to make timeconsuming adjustments on the instrument. The technique is also excellent for stereo microscopy. This method was inspired by a more elaborate method described by MacGillivary and Ehrman [2]. This method is fast, simple, and inexpensive and can be used by almost any facility for randomly orienting diatoms.

Adding diatoms to SEM stubs can follow a number of procedures, most of which involve pipetting whole cells cleaned of organics onto glass coverslips, carbon stickies, filter papers, or membrane filters, which are then attached to the stub [3-8]. Alternatively, and more precisely, diatoms can be added directly to an aluminum stub [4]. For SEM examination of diatoms, prepared aluminum stubs are normally sputter coated with a sufficient thickness (often 8-15 $\mathrm{nm})$ of gold or gold/palladium ( $\mathrm{Au} / \mathrm{Pd})$ alloy to increase the signal to noise ratio and to suppress charging.

\section{Materials and Methods}

Making grooves in SEM stubs. Initially, a $12.7 \mathrm{~mm}$ aluminum SEM stub was finely sanded of all roughness using
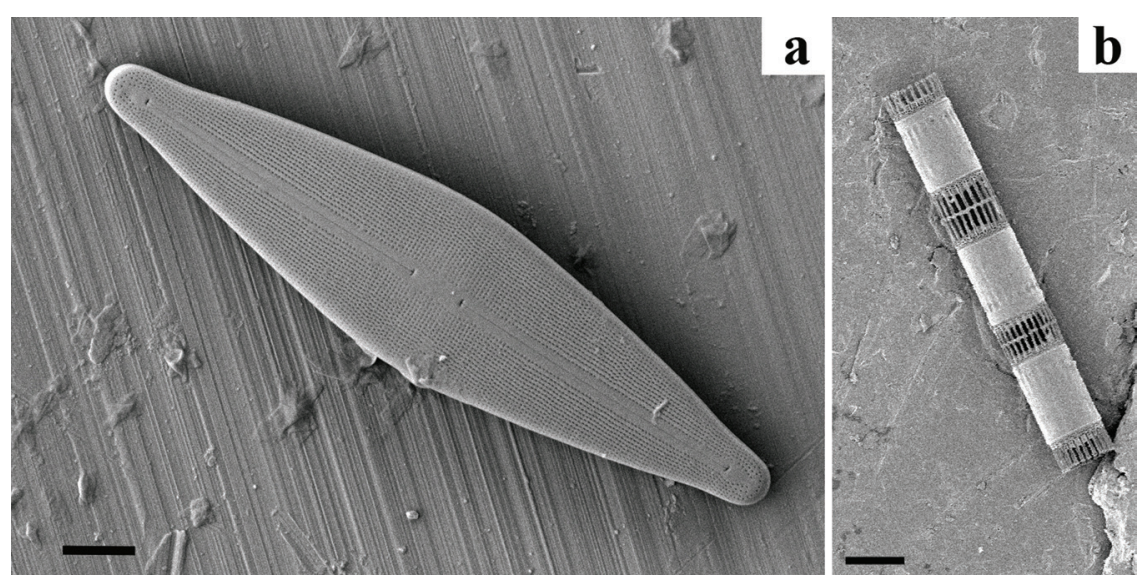

Figure 1: Images illustrating the limitations of examining diatoms lying flat on an un-grooved aluminum stub. (a) A pennate freshwater diatom naturally oriented onto the valve face. The girdle morphology is not clearly visible even with fully tilting the SEM stage. (b) A colony of the marine diatom Skeletonema sp. in typical girdle facing orientation. Even with tilting of the stage, a clear view of the valve cannot be obtained. Bars in $(a)=5 \mu \mathrm{m},(b)=10 \mu \mathrm{m}$. 

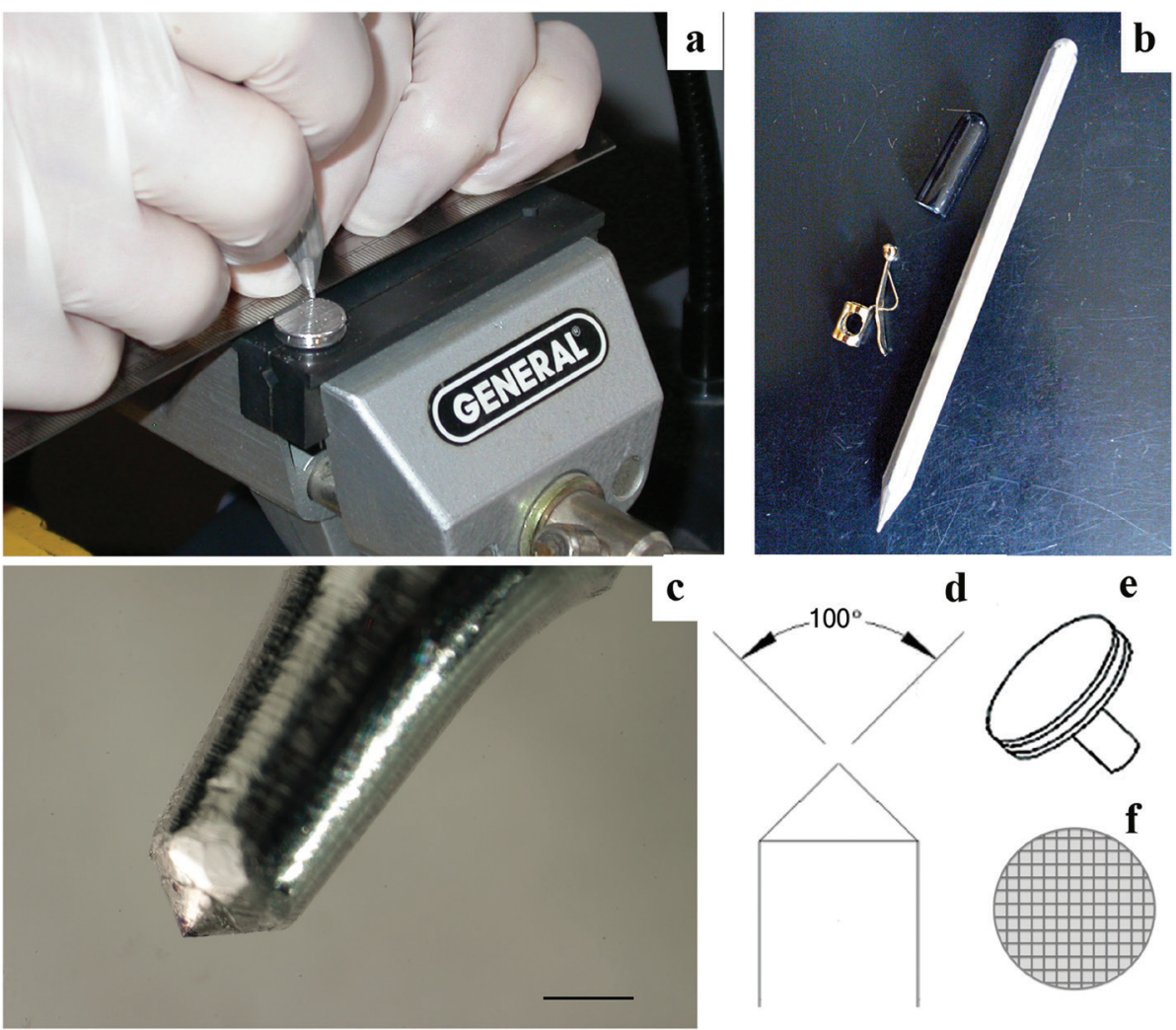

d

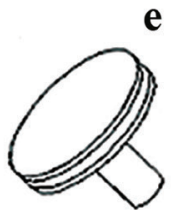

Fure 2: Making grooves in aluminum SEM stubs. (a) Vise, stub, metal rule, and scoring to make grooves. (b) Diamond scribe and (c) tip of scribe. (d) Angle of tip and (e) a typical aluminum SEM stub. (f) Groove pattern.
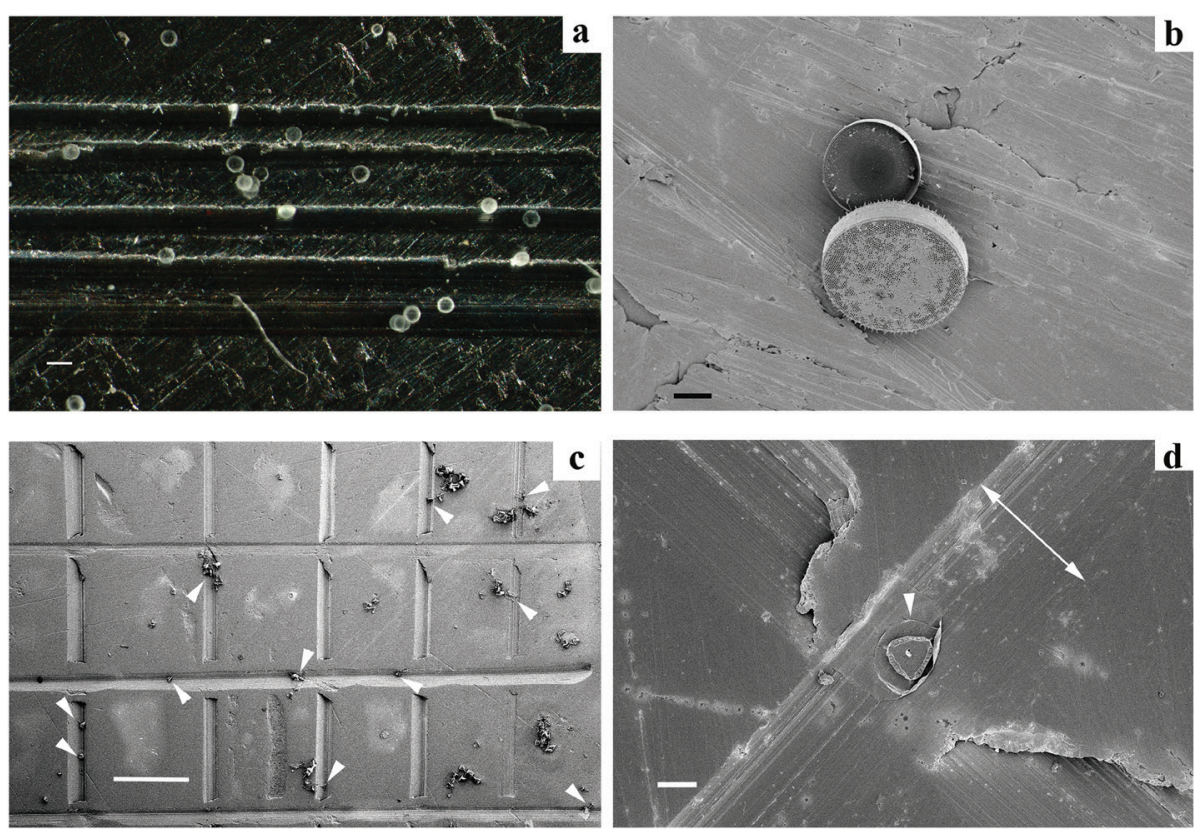

Figure 3: (a) Cells of Thalassiosira punctigera strewn on a SEM stub with grooves. (b) No tilting of the SEM stage. In the center of Figure 3a, there are two diatoms of T. punctigera. (c) Ditylum brightwellii cells (arrows) on grids spaced approximately $200-800 \mu \mathrm{m}$ apart and $70-125 \mu \mathrm{m}$ wide. No tilting of the SEM stage. (d) A cell of $D$. brightwellii, from the stub in Figure (c) that fell into a groove valve face upwards. The single arrowhead indicates the location of the cell. The double arrow shows the width of the groove, which is $73 \mu \mathrm{m}$ wide. Bars in $(\mathrm{a})=75 \mu \mathrm{m}$, in $(\mathrm{b})=20 \mu \mathrm{m}$, in $(\mathrm{c})=500 \mu \mathrm{m}$, in $(\mathrm{d})=20 \mu \mathrm{m}$.
1200 grit silicon carbide paper, wetted, on a hard, flat surface. The debris was cleaned off with double distilled water (DDW). Then the stub was securely fastened in a vise mounted on a bench (Figure 2a). A steel rule was used as a guide to score deep grooves by hand pressure using a diamond scribe (Figures. $2 \mathrm{a}-2 \mathrm{c})$ at a measured angle of approximately $100^{\circ}$ on standard aluminum stubs (Figure $2 \mathrm{~d}-\mathrm{e}$ ). The ruler was then placed at $90^{\circ}$ to the previous grooves to produce a grid pattern (Figure 2f). A little practice was required to make approximately evenly spaced grooves between $20 \mu \mathrm{m}$ and $125 \mu \mathrm{m}$ wide and about $1 \mathrm{~mm}$ or less apart. Holding the scribe vertically resulted in even, deep grooves. Experimenting with scribing pressure allowed for an optimal groove width for the size of diatoms being studied. Again, with wetted 1200 silicon carbide paper, the stub was gently and quickly sanded to remove high points caused by the scribe. The stubs were cleaned by brush scrubbing with soap and water and rinsed well in DDW. After drying, stubs were polished on a folded paper towel backed by a hard surface, or polished to 14,000 grit (1 to $2 \mu \mathrm{m}$ ) with a wet diamond wheel or sheet, then finally cleaned with 100\% ethyl alcohol. With a little practice, it took 6-10 minutes to make each stub.

Cell deposition procedure. Generally, frustules are cleaned with either acids or hydrogen peroxide [4]. Different types of diatoms usually require different cleaning methods. The robust Coscinodiscus are cleaned with a different method than delicate Ditylum spp. After using an applicable cleaning method for the sample being investigated, the salts used in cleaning are washed out with DDW, centrifuging between washing, depending on cleaning method and type of diatom. Household bleach tends to produce a lot of salts appearing on stubs and on the specimens. So to be safe, especially for SEM, wash with DDW at least $100 \mathrm{~mL}$, even better with $150 \mathrm{ml}$. If using hydrogen peroxide then less salts and less washing with DDW. With a delicate diatom like Ditylum, it is best to let it settle for over $2 \mathrm{hr}$., or centrifuge at $1600 \mathrm{rpm}$ for 30-40 min. More robust diatoms like Coscinodiscus can handle $3000 \mathrm{rpm}$ for $30 \mathrm{~min}$. The frustules settle to the bottom of tubes very quickly, most likely 


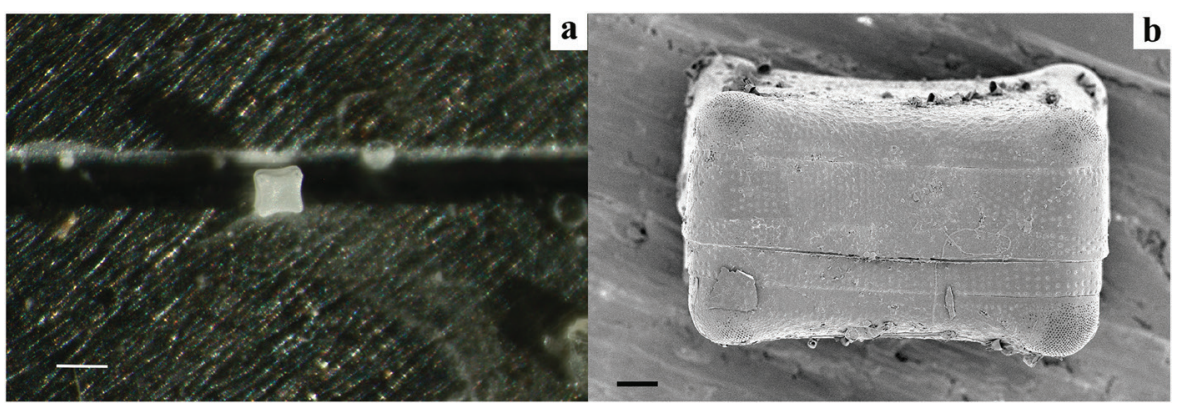

Figure 4: (a) The centric diatom Biddulphia antediluviana, oriented in both valve and girdle views (stereo micrograph). (b) SEM of $B$. antediluviana in a groove, girdle view. Bars in (a) $=75 \mu \mathrm{m}$, in (b)=10 $\mu \mathrm{m}$.
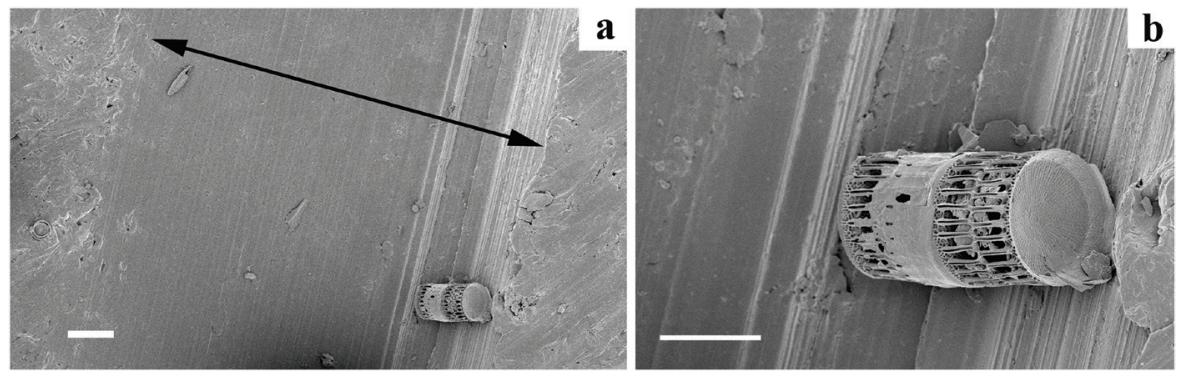

Figure 5: (a-b) The marine diatom Skeletonema sp. in a groove in a valve-up orientation, also showing the valve view. Black arrow showing the width of the groove is $176 \mu \mathrm{m}$. Bars in (a) $=20 \mu \mathrm{m}$, in (b) $=10 \mu \mathrm{m}$.

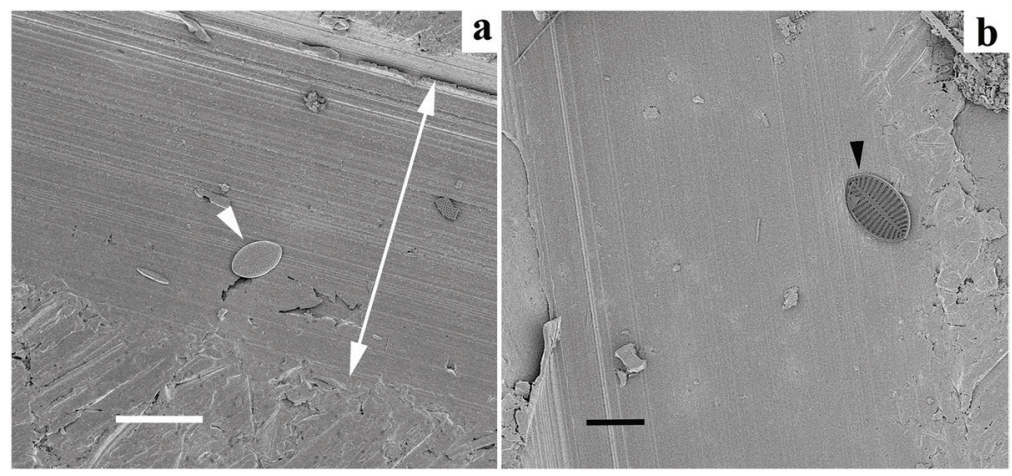

Figure 6: (a) The diatom Cocconeis sp. laying on a groove, valve face up. (b) Internal view of a Cocconeis $s p$. valve. Minimal tilting of the SEM stage is required. Bars $=20 \mu \mathrm{m}$.
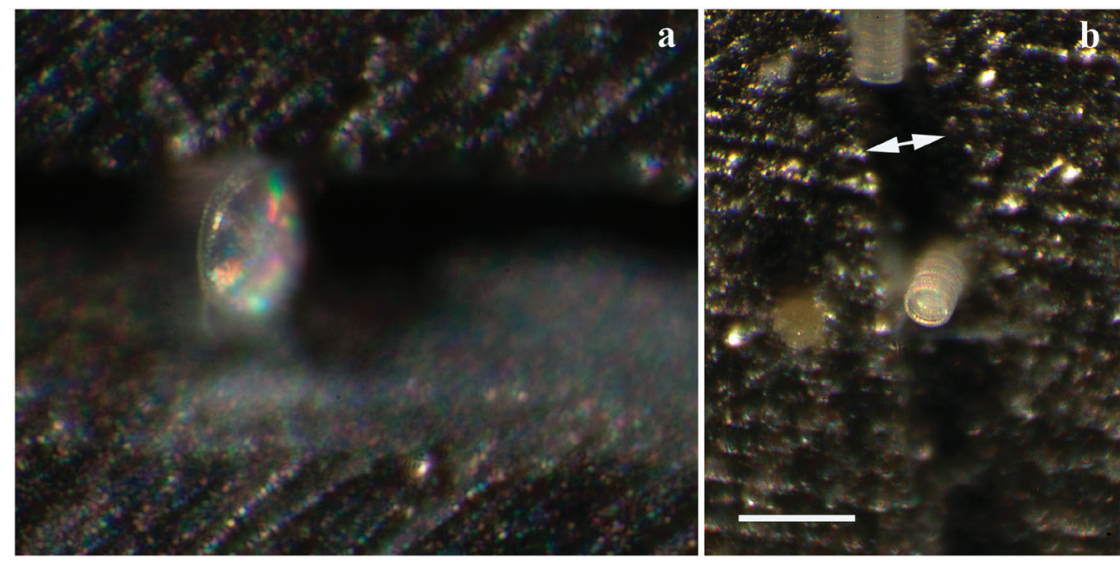

Figure 7: (a) A centric diatom, Thalassiosira sp., oriented so the girdle is in view: a non-typical orientation. (b) The marine diatom Paralia sulcata, lying in a groove (double arrow), both in girdle and valve orientations. Both are images of cleaned diatoms, taken with a Leica S8APO stereo microscope using side lighting. Bar $=100 \mu \mathrm{m}$. within $10-15$ min. Centrifuging quickens the process. A more detailed review of cleaning methods is in preparation.

The cells were then re-suspended in either DDW or an ethanol/DDW mixture (50-80\% ethanol) and micro-pipetted directly onto the aluminum stubs. The advantage of using the ethanol mixture was rapid evaporation to reduce drying time of the samples before sputter coating, and movement of the cells as the ethanol dried allowed for a greater chance of random orientation and less clumping and piling of cells, one on top of another. It also effectively reduces drying time of the stub before SEM observation, often eliminating the use of an oven. After air-drying (either overnight or for $10-15 \mathrm{~min}$. in a $60^{\circ} \mathrm{C}$ oven), the cell density and distribution was checked with a stereo microscope to systematically observe the number of cells and to make sure the necessary orientations obtained for SEM imaging were available. There needs to be enough cells as a single layer but not overlapping each other. Once accepted, samples were sputter coated with 8-15 nm Au/Pd for viewing on the SEM.

Imaging. All SEM images were taken with the Hitachi S-4800 FESEM at the Advanced Microscope Facility, University of Victoria, BC Canada, except Figure 1a, which was imaged on a JEOL $6700 \mathrm{~F}$ FESEM, Microscopy Otago, University of Otago, Dunedin, New Zealand. LM images were taken with a Leica S8APO Stereomicroscope with a Canon Rebel EOS T1i digital camera with a Martin Microscopes MM SLR adaptor.

\section{Results}

Producing a gridded pattern on the stub surface increased the number of grooves per stub, thereby increasing favorable orientations available for imaging (Figures 3-7). This method allowed for many diatoms to randomly orient so surfaces and features, especially internal structures that are normally obscured using a flat surface, were advantageously viewed by SEM and LM. Less search time was required to find cells in different orientations, and less time was spent rotating and tilting the SEM stage, which often meant less need to adjust beam alignment if the stage had been tilted.

\section{Conclusions}

Creating grooves in a grid pattern on an SEM stub in this manner is an effective, 
simple, quick, and inexpensive method for producing a greater range of orientations of diatom frustules and valves compared with using a flat mount.

\section{Acknowledgements}

Our thanks to Rafael Hoekstra and Arjan van Asselt for lab assistance, Liz Girvan for SEM technical support at the University of Otago, New Zealand, and support from the Advanced Microscopy Facility at the University of Victoria, Canada.

\section{References}

[1] M Hildebrand, "Marine Diatoms and Their Role in the Ecosystem," https://aaas.confex.com/aaas/2010/webprogram/Paper1743.html.

[2] ML MacGillivary and JM Ehrman, Diatom Research 26(4) (2011) 321-23.

[3] FE Round, Annals of Botany 53 (1984) 457-68.

[4] F Round et al., The Diatoms, Biology \& Morphology of the Genera, Cambridge University Press, Cambridge, UK, 1990.

[5] GR Hasle and EE Syvertsen, "Marine Diatoms" in Identifying Marine Phytoplankton, ed. CR Tomas, Academic Press, San Diego, 1997, pp. 334-36.

[6] J Pickett-Heaps, J Phycol 34 (1998) 1088-89.

[7] I Jameson and GM Hallegraeff, Algae of Australia. Phytoplankton of temperate waters, eds. GM Hallegraeff et al., Canberra \& Melbourne: ABRS; CSIRO Publishing, Clayton, Australia, 2010, pp. 16-82.

[8] SS Lee et al., Diatom Research 29(4) (2014) 325-50.

\section{MRS-6}

We are ISO-9000 certified and ISO-17025 accredited Microscopy Calibration Standard

Now you can calibrate better from $1,000 X$ to $1,000,000 X$ !

This is our fifth generation, traceable, magnification reference standard for all types (SEM, FESEM, Optical, STM, AFM, etc.) of microscopy. The MRS- 6 has multiple $X$ and $Y$ pitch patterns ranging from $80 \mathrm{~nm}( \pm 3 \mathrm{~nm})$ to $2 \mu \mathrm{m}$ and $3 \mathrm{bar}$ targets from $80 \mathrm{~nm}$ to $3 \mu \mathrm{m}$. There is also a STM test pattern. Definition of the $80 \mathrm{~nm}$ pitch pattern is excellent.

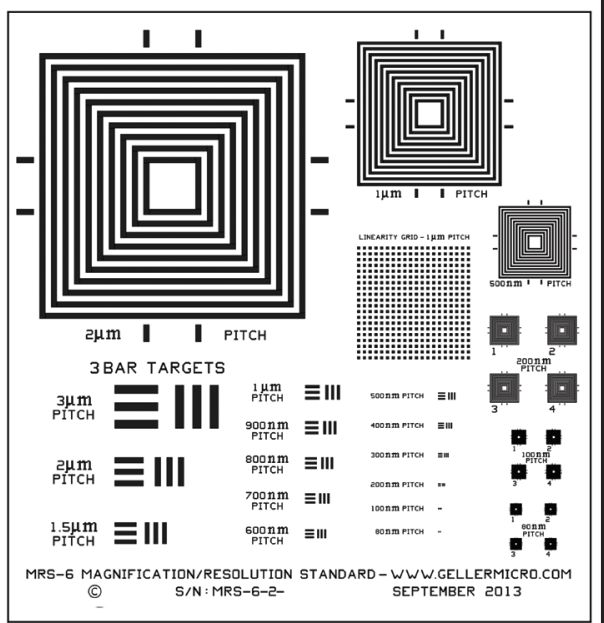

\section{GELLER}

MICROANALYTICAL LABORATORY, Inc.

426e BOSTON ST., TOPSFIELD, MA 01983-1216 TEL: 978 887-7000 FAX: 978-887-6671 www.GellerMicro.com

Don't Let a Fingerprint Ruin Your Day-Use Evactron ${ }^{\circledR}$ Cleaning

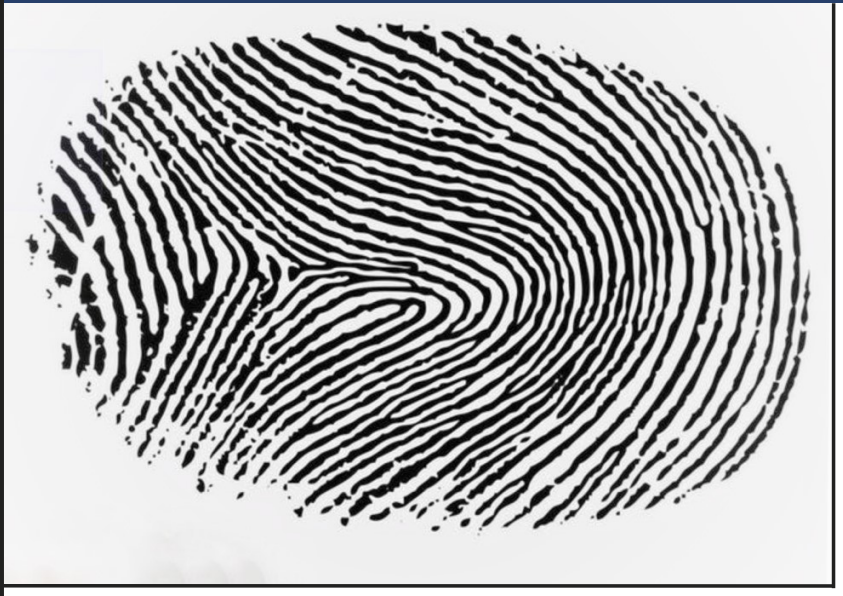

Fingerprints are one of the major sources of contamination in vacuum systems. Evactron dual-action turbo plasma cleaning ${ }^{T M}$ expels these adventitious hydrocarbons with:

* Oxygen radicals plus UV active desorption

* Hollow cathode plasma radical source

* No kinetic sputter etch damage or debris

* A compact and efficient plasma source

* Cleaning in minutes for days of perfect imaging and analysis

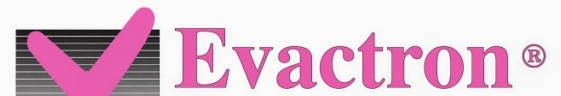

By XEI Scientific
Need clean samples and chamber surfaces? Let us show you the "fastest way to pristine"тм!

WWW.EVACTRON.COM 1-650-369-0133 